\title{
Primordial helium abundance of the SMC: a view from intermediate mass stars
}

\author{
Roberto D. D. Costa ${ }^{1}$, Walter J. Maciel ${ }^{1}$ and Thais E. P. Idiart ${ }^{1}$ \\ ${ }^{1}$ IAG, University of São Paulo, \\ Rua do Matão 1226, 05508-090, São Paulo/SP, Brazil \\ email: roberto@astro.iag.usp.br, maciel@astro.iag.usp.br, thais@astro.iag.usp.br
}

\begin{abstract}
Helium abundances were derived for a sample of planetary nebulae of the Small Magellanic Cloud. These abundances were corrected from the chemical evolution of the galaxy as well as from stellar nucleosynthesis, and the primordial helium abundance then was estimated for the sample. Results indicate that the resulting average value for the sample is consistent with the expected values for primordial helium from SBBN and other values derived from HII regions in different stellar systems, even varying the enrichment ratio within its uncertainty range.
\end{abstract}

Keywords. ISM: planetary nebulae; stars: abundances; galaxies: Magellanic Clouds

\section{Introduction}

We derived the chemical composition for a sample of planetary nebulae of the Small Magellanic Cloud. Helium abundances were estimated as accurately as possible, including correction of collisional effects using collision-to-recombination correction factors. Based on luminosities and effective temperatures derived for the central star of each nebula, the masses, and then ages of the progenitor stars were estimated using isochrones and massage relationships. Using these results, helium abundance for each nebula was corrected for the contamination from the evolution of their progenitor star as well as from the chemical evolution of the interstellar medium, by using yields for intermediate mass stars and a helium-to-heavy elements abundance ratio. Therefore we determined the helium enrichment in the SMC and used these results to estimate the pregalactic helium abundance of this galaxy.

\section{Results and discussion}

The observational sample consisted in $49 \mathrm{PNe}$ of the SMC. Observations were made at ESO/La Silla and OPD/Brazil. The observation and analysis procedures are described by Idiart et al. (2007). Primordial helium was estimated for each nebula using the expression

$$
Y_{P}=Y-\frac{\Delta Y}{\Delta Z} Z-\Delta Y_{S}
$$

where $Y$ was derived from the helium abundance, $Z$ was estimated from the chemical abundances, $\Delta Y_{S}$ are the helium yields from van den Hoek \& Groenewegen (1997) and $\Delta Y / \Delta Z=2.0 \pm 0.6$ was taken from Peimbert et al. (2007).

The results are in Table 1, that shows the average values for primordial helium derived from the expression above, using the described PNe sample and excluding objects with neutral helium, without available yield and type-I PNe. The primordial abundances were calculated for $\Delta Y / \Delta Z=2.0 \pm 0.6$. The error is mainly due to uncertainties in the mass determination of the progenitor stars, that were derived from their effective temperatures and luminosities using isochrones and mass-age relationships. 
Table 1. Average values for the primordial helium abundance.

\begin{tabular}{ccc}
\hline$\Delta Y / \Delta Z$ & $\overline{Y_{P}}$ & Error \\
\hline 2.0 & 0.234 & 0.009 \\
2.6 & 0.234 & 0.009 \\
1.4 & 0.241 & 0.008 \\
\hline
\end{tabular}

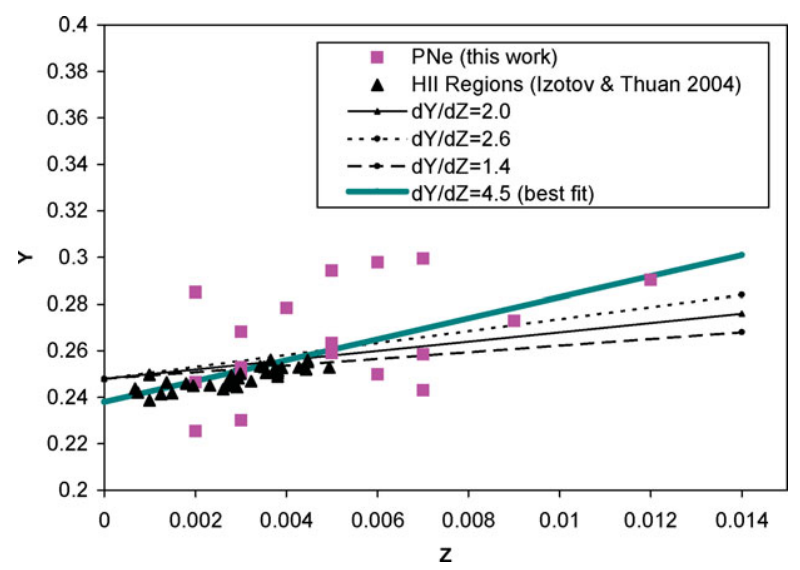

Figure 1. Y vs Z relation derived from our data, compared to a sample of HII regions in blue compact galaxies.

Figure 1 shows the $\mathrm{Y}$ vs $\mathrm{Z}$ relation derived from our data, compared to a sample of HII regions in blue compact galaxies, that are usually adopted to derive primordial helium abundances, taken from Izotov \& Thuan (2004). It can be seen that, despite their larger dispersion, the data from PNe fit well the abundance distribution of HII regions. The thin lines correspond to $\Delta Y / \Delta Z=2.0 \pm 0.6$, using the theoretical value of $Y_{P}=0.248$ (Steigman 2007) as the $Z=0$ value. The thick line corresponds to the best fit for both samples combined, which resulted in $\Delta Y / \Delta Z=4.5$. It is important to note that this steeper value is consistent with that reported by Izotov \& Thuan (2004): $\Delta Y / \Delta Z=3.7 \pm 1.2$. The figure also shows that, despite their different $\mathrm{Z}$ ranges, enrichment ratios derived from HII regions and $\mathrm{PNe}$ are compatible.

From this result it is possible to see that $\mathrm{He} / \mathrm{H}$ abundances derived from planetary nebulae can be used to study helium abundances throughout a stellar system like the SMC, and the resulting $Y_{P}$ values calculated as described above are consistent with the expected values for primordial helium from SBBN and other values derived from HII regions in different stellar systems, even varying the enrichment ratio within its uncertainty range. Moreover, our results indicate that despite their metallicities are distributed in different $\mathrm{Z}$ ranges, enrichment ratios of HII regions and $\mathrm{PNe}$ are compatible, although a larger sample of PNe is required to improve these results.

\section{References}

Idiart, T. P., Maciel, W. J., \& Costa, R. D. D. 2007, A\&A, 472, 101

Izotov, Y. I., Thuan, T. X. 2004, ApJ, 602, 200

Peimbert, M., Luridiana, V., Peimbert, A., \& Carigi, L. 2007, ASPC, 374, 81

Steigman, G. 2007, Ann.Rev.Nuc.Part.Sci. 57, 463

van den Hoek, L. B., \& Groenewegen, M. A. T. 1997, A\&AS, 123, 305 